\title{
Dirac Quasi-Normal Modes in Schwarzschild Black Hole Spacetimes
}

\author{
H. T. Chd* \\ Department of Physics, Tamkang University, \\ Tamsui, Taipei, Taiwan, Republic of China
}

(Dated: October 23, 2018)

\begin{abstract}
We evaluate both the massless and the massive Dirac quasi-normal mode frequencies in the Schwarzschild black hole spacetime using the WKB approximation. For the massless case, we find that, similar to those for the integral spin fields, the real parts of the frequencies increase with the angular momentum number $\kappa$, while the imaginary parts or the dampings increase with the mode number $n$ for fixed $\kappa$. For the massive case, the oscillation frequencies increase with the mass $m$ of the field, while the dampings decrease. Fields with higher masses will therefore decay more slowly.
\end{abstract}

\footnotetext{
*Electronic address: htcho@mail.tku.edu.tw
} 


\section{INTRODUCTION}

In a series of papers, Finster and collaborators [1, 2, 3, 4, 5, 6, 7, 8, 9, 10] studied the interaction of the Dirac field with gravity. While they have found stable particlelike solutions in the Einstein-Dirac-Maxwell system [1, 2], they have also proved the non-existence of time-periodic solutions in various black hole spacetimes [4, 5]. This is quite surprising because it means that Dirac particles, including electrons and neutrinos, cannot remain on a periodic orbit around a black hole. In [3], they also showed that there are no spherically symmetric black hole solutions in the Einstein-Dirac-Maxwell system other than the Reissner-Nordström one. This indicates that if a "cloud" of Dirac particles collapses gravitationally, the particles must eventually vanish inside the event horizon of a black hole or escape to infinity. It is therefore interesting to see how Dirac fields evolve in curved background spacetimes. To understand this, Finster and collaborators considered the massive Dirac field in the general Newman-Kerr black hole spacetime and derived the so-called power-law tail behavior of the field at very late time in [9, 10].

The evolution of wave fields around a black hole consists roughly of three stages [11]. The first one is an initial wave burst coming directly from the source and is dependent on the initial form of the original wave field. The second one involves the damped oscillations called the quasi-normal modes, which do not depend on the initial values of the wave but are characteristic of the background black hole spacetimes. The normal mode frequencies have complex values because of radiation damping. The last stage is the power-law tail behavior mentioned above. It arises because of the backscattering by the long-range gravitational field.

Here we would like to concentrate on the intermediate stage of the evolution of the Dirac field where the quasi-normal modes dominate. As a first step we would calculate the massive Dirac quasi-normal mode frequencies in the Schwarzschild black hole spacetime in this paper. The study of black hole quasi-normal modes has a long history [12]. It started with the black hole stability problem which concerned the evolutions of black hole perturbations. Since these perturbations are effectively wave fields of spins 0,1 , and 2 , the evaluations of the quasi-normal mode frequencies are mainly done for these cases. As far as the Dirac field is concerned, we are not aware of any work done in this direction. So we hope that our calculation can serve to fill in this gap. 
There are quite a few methods in evaluating the quasi-normal mode frequencies. Most of them are numerical in nature. However, a powerful analytical WKB scheme was devised by Schutz and Will [13], and was subsequently extended to higher orders in 14]. Comparing with other approaches this scheme is shown to be accurate for the low-lying modes [15]. Since we are interested in the evolutions of the Dirac fields in the intermediate stage, which is dominated by the low-lying modes, this WKB scheme is good enough for our purpose.

In the next section, we consider the Dirac equation in the Schwarzschild spacetime and its reduction into a set of Schrödinger-like equations with a particular effective potential. In Section III, we evaluate the quasi-normal frequencies using the WKB scheme for the massless case. The massive case is considered in Section IV. Conclusions and discussions are presented in Section V.

\section{DIRAC EQUATION IN THE SCHWARZSCHILD SPACETIME}

Consider the Dirac equation in a general background spacetime [16],

$$
\left[\gamma^{a} e_{a}{ }^{\mu}\left(\partial_{\mu}+\Gamma_{\mu}\right)+m\right] \Psi=0
$$

where $m$ is the mass of the Dirac field, and $e_{a}{ }^{\mu}$ is the inverse of the tetrad $e_{\mu}{ }^{a}$ defined by the metric $g_{\mu \nu}$,

$$
g_{\mu \nu}=\eta_{a b} e_{\mu}^{a} e_{\nu}^{b}
$$

with $\eta_{a b}=\operatorname{diag}(-1,1,1,1)$ being the Minkowski metric. $\gamma^{a}$ are the Dirac matrices

$$
\gamma^{0}=\left(\begin{array}{cc}
-i & 0 \\
0 & i
\end{array}\right), \quad \gamma^{i}=\left(\begin{array}{cc}
0 & -i \sigma^{i} \\
i \sigma^{i} & 0
\end{array}\right), i=1,2,3
$$

where $\sigma^{i}$ are the Pauli matrices. $\Gamma_{\mu}$ is the spin connection given by

$$
\Gamma_{\mu}=\frac{1}{8}\left[\gamma^{a}, \gamma^{b}\right] e_{a}^{\nu} e_{b \nu ; \mu}
$$

where $e_{b \nu ; \mu}=\partial_{\mu} e_{b \nu}-\Gamma_{\mu \nu}^{\alpha} e_{b \alpha}$ is the covariant derivative of $e_{b \nu}$ with $\Gamma_{\mu \nu}^{\alpha}$ being the Christoffel symbols.

In the Schwarzschild spacetime,

$$
d s^{2}=-\left(1-\frac{2 M}{r}\right) d t^{2}+\left(1-\frac{2 M}{r}\right)^{-1} d r^{2}+r^{2} d \theta^{2}+r^{2} \sin ^{2} \theta d \phi^{2}
$$


where $M$ is the mass of the black hole. Here we can take the tetrad to be

$$
e_{\mu}^{a}=\operatorname{diag}\left(\left(1-\frac{2 M}{r}\right)^{1 / 2},\left(1-\frac{2 M}{r}\right)^{-1 / 2}, r, r \sin \theta\right) .
$$

Then the spin connection defined by Eq. (4) is

$$
\gamma^{a} e_{a}{ }^{\mu} \Gamma_{\mu}=\gamma^{1}\left(1-\frac{2 M}{r}\right)^{1 / 2}\left(\frac{1}{r}+\frac{M}{2 r(r-2 M)}\right)+\gamma^{2}\left(\frac{1}{2 r}\right) \cot \theta .
$$

Therefore, the Dirac equation becomes

$$
\begin{aligned}
& {\left[\gamma^{0}\left(1-\frac{2 M}{r}\right)^{-1 / 2} \frac{\partial}{\partial t}+\gamma^{1}\left(1-\frac{2 M}{r}\right)^{1 / 2}\left(\frac{\partial}{\partial r}+\frac{1}{r}+\frac{M}{2 r(r-2 M)}\right)\right.} \\
& \left.\quad+\gamma^{2}\left(\frac{1}{r}\right)\left(\frac{\partial}{\partial \theta}+\frac{1}{2} \cot \theta\right)+\gamma^{3}\left(\frac{1}{r \sin \theta}\right) \frac{\partial}{\partial \phi}+m\right] \Psi=0 .
\end{aligned}
$$

The equation can be simplified by defining

$$
\Psi=\left(1-\frac{2 M}{r}\right)^{-1 / 4} \Phi
$$

Then Eq. (8) becomes

$$
\begin{aligned}
& {\left[\gamma^{0}\left(1-\frac{2 M}{r}\right)^{-1 / 2} \frac{\partial}{\partial t}+\gamma^{1}\left(1-\frac{2 M}{r}\right)^{1 / 2}\left(\frac{\partial}{\partial r}+\frac{1}{r}\right)\right.} \\
& \left.\quad+\gamma^{2}\left(\frac{1}{r}\right)\left(\frac{\partial}{\partial \theta}+\frac{1}{2} \cot \theta\right)+\gamma^{3}\left(\frac{1}{r \sin \theta}\right) \frac{\partial}{\partial \phi}+m\right] \Phi=0
\end{aligned}
$$

which is closely related to the Dirac equation in flat spacetime with a central potential [17]. We can try the ansatz,

$$
\Phi(t, r, \theta, \phi)=\left(\begin{array}{c}
\frac{i G^{( \pm)}(r)}{r} \varphi_{j m}^{( \pm)}(\theta, \phi) \\
\frac{F^{( \pm)}(r)}{r} \varphi_{j m}^{(\mp)}(\theta, \phi)
\end{array}\right) e^{-i E t}
$$

where for $j=l+1 / 2$,

$$
\varphi_{j m}^{(+)}=\left(\begin{array}{c}
\sqrt{\frac{l+1 / 2+m}{2 l+1}} Y_{l}^{m-1 / 2} \\
\sqrt{\frac{l+1 / 2-m}{2 l+1}} Y_{l}^{m+1 / 2}
\end{array}\right)
$$

and for $j=l-1 / 2$,

$$
\varphi_{j m}^{(-)}=\left(\begin{array}{c}
\sqrt{\frac{l+1 / 2-m}{2 l+1}} Y_{l}^{m-1 / 2} \\
-\sqrt{\frac{l+1 / 2+m}{2 l+1}} Y_{l}^{m+1 / 2}
\end{array}\right) .
$$

Then the radial equations for $F^{( \pm)}$and $G^{( \pm)}$can be simplified to

$$
\frac{d}{d r_{*}}\left(\begin{array}{c}
F^{( \pm)} \\
G^{( \pm)}
\end{array}\right)-\sqrt{1-\frac{2 M}{r}}\left(\begin{array}{cc}
\kappa_{( \pm)} / r & m \\
m & -\kappa_{( \pm)} / r
\end{array}\right)\left(\begin{array}{c}
F^{( \pm)} \\
G^{( \pm)}
\end{array}\right)=\left(\begin{array}{cc}
0 & -E \\
E & 0
\end{array}\right)\left(\begin{array}{c}
F^{( \pm)} \\
G^{( \pm)}
\end{array}\right),
$$


where we have made a coordinate change

$$
r_{*}=r+2 M \ln \left(\frac{r}{2 M}-1\right),
$$

and the constant

$$
\kappa_{( \pm)}=\left\{\begin{array}{cc}
-(j+1 / 2), & j=l+1 / 2 \\
(j+1 / 2), & j=l-1 / 2
\end{array}\right.
$$

Note that $\kappa_{(+)}$and $\kappa_{(-)}$are negative and positive integers, respectively.

To further simplify the radial equations, we consider separately the $(+)$ and $(-)$ cases. First, for $(+)$, we make a change of variables [18]

$$
\left(\begin{array}{c}
\hat{F}^{(+)} \\
\hat{G}^{(+)}
\end{array}\right)=\left(\begin{array}{cc}
\sin \left(\theta_{(+)} / 2\right) & \cos \left(\theta_{(+)} / 2\right) \\
\cos \left(\theta_{(+)} / 2\right) & -\sin \left(\theta_{(+)} / 2\right)
\end{array}\right)\left(\begin{array}{c}
F^{(+)} \\
G^{(+)}
\end{array}\right),
$$

where

$$
\theta_{(+)}=\tan ^{-1}\left(m r /\left|\kappa_{(+)}\right|\right) .
$$

Then Eq. (14) becomes

$$
\begin{aligned}
& \frac{d}{d r_{*}}\left(\begin{array}{c}
\hat{F}^{(+)} \\
\hat{G}^{(+)}
\end{array}\right)-\sqrt{1-\frac{2 M}{r}} \sqrt{\left(\frac{\left.\kappa_{(+)}\right)^{2}+m^{2}}{r}\left(\begin{array}{cc}
1 & 0 \\
0 & -1
\end{array}\right)\left(\begin{array}{c}
\hat{F}^{(+)} \\
\hat{G}^{(+)}
\end{array}\right)\right.} \\
& \quad=-E\left(1+\frac{1}{2 E}\left(1-\frac{2 M}{r}\right) \frac{m\left|\kappa_{(+)}\right|}{\kappa_{(+)}^{2}+m^{2} r^{2}}\right)\left(\begin{array}{cc}
0 & -1 \\
1 & 0
\end{array}\right)\left(\begin{array}{c}
\hat{F}^{(+)} \\
\hat{G}^{(+)}
\end{array}\right) .
\end{aligned}
$$

This equation can be simplified further if we make yet another change of variable,

$$
\hat{r}_{*}=r_{*}+\frac{1}{2 E} \tan ^{-1}\left(\frac{m r}{\left|\kappa_{(+)}\right|}\right),
$$

and then

$$
\frac{d}{d \hat{r}_{*}}\left(\begin{array}{c}
\hat{F}^{(+)} \\
\hat{G}^{(+)}
\end{array}\right)+W_{(+)}\left(\begin{array}{c}
-\hat{F}^{(+)} \\
\hat{G}^{(+)}
\end{array}\right)=E\left(\begin{array}{c}
\hat{G}^{(+)} \\
-\hat{F}^{(+)}
\end{array}\right),
$$

where

$$
W_{(+)}=\frac{\sqrt{1-\frac{2 M}{r}} \sqrt{\left(\frac{\kappa_{(+)}}{r}\right)^{2}+m^{2}}}{1+\frac{1}{2 E}\left(1-\frac{2 M}{r}\right)\left(\frac{m\left|\kappa_{(+)}\right|}{\kappa_{(+)}^{2}+m^{2} r^{2}}\right)} .
$$

These equations for $\hat{F}^{(+)}$and $\hat{G}^{(+)}$can be decoupled giving

$$
\begin{aligned}
& \left(-\frac{d^{2}}{d \hat{r}_{*}^{2}}+V_{(+) 1}\right) \hat{F}^{(+)}=E^{2} \hat{F}^{(+)}, \\
& \left(-\frac{d^{2}}{d \hat{r}_{*}^{2}}+V_{(+) 2}\right) \hat{G}^{(+)}=E^{2} \hat{G}^{(+)},
\end{aligned}
$$


where

$$
V_{(+) 1,2}= \pm \frac{d W_{(+)}}{d \hat{r}_{*}}+W_{(+)}^{2}
$$

For the case of $(-)$, similar procedures can be carried out giving

$$
\begin{aligned}
& \left(-\frac{d^{2}}{d \hat{r}_{*}^{2}}+V_{(-) 1}\right) \hat{F}^{(-)}=E^{2} \hat{F}^{(-)}, \\
& \left(-\frac{d^{2}}{d \hat{r}_{*}^{2}}+V_{(-) 2}\right) \hat{G}^{(-)}=E^{2} \hat{G}^{(-)},
\end{aligned}
$$

where

$$
V_{(-) 1,2}= \pm \frac{d W_{(-)}}{d \hat{r}_{*}}+W_{(-)}^{2}
$$

with

$$
W_{(-)}=\frac{\sqrt{1-\frac{2 M}{r}} \sqrt{\left(\frac{\kappa_{(-)}}{r}\right)^{2}+m^{2}}}{1-\frac{1}{2 E}\left(1-\frac{2 M}{r}\right)\left(\frac{m \kappa_{(-)}}{\kappa_{(-)}^{2}+m^{2} r^{2}}\right)} .
$$

From the forms of $W_{( \pm)}$in Eqs. (22) and (29), we see that the $(+)$and $(-)$cases can be put together giving

$$
\begin{aligned}
& \left(-\frac{d^{2}}{d \hat{r}_{*}^{2}}+V_{1}\right) \hat{F}=E^{2} \hat{F}, \\
& \left(-\frac{d^{2}}{d \hat{r}_{*}^{2}}+V_{2}\right) \hat{G}=E^{2} \hat{G},
\end{aligned}
$$

where

$$
V_{1,2}= \pm \frac{d W}{d \hat{r}_{*}}+W^{2}
$$

with

$$
\begin{aligned}
W & =\frac{\sqrt{1-\frac{2 M}{r}} \sqrt{\left(\frac{\kappa}{r}\right)^{2}+m^{2}}}{1+\frac{1}{2 E}\left(1-\frac{2 M}{r}\right)\left(\frac{m \kappa}{\kappa^{2}+m^{2} r^{2}}\right)} \\
& =\frac{\Delta^{1 / 2}\left(\kappa^{2}+m^{2} r^{2}\right)^{3 / 2}}{r^{2}\left(\kappa^{2}+m^{2} r^{2}\right)+m \kappa \Delta / 2 E},
\end{aligned}
$$

where $\Delta=r(r-2 M)$. Here $\kappa$ goes over all positive and negative integers. Positive integers represent the $(+)$ case with

$$
\kappa=j+\frac{1}{2} \quad \text { and } \quad j=l+\frac{1}{2}
$$

while negative integers represent the $(-)$ case with

$$
\kappa=-\left(j+\frac{1}{2}\right) \quad \text { and } \quad j=l-\frac{1}{2}
$$


From the Schrödinger-like equations in Eqs. (30) and (31), we shall evaluate the corresponding quasi-normal mode frequencies. Note that $V_{1}$ and $V_{2}$, which are related as shown in Eq. (32), are supersymmetric partners derived from the same superpotential $W$ [19]. It has been shown that potentials related in this way possess the same spectra of quasi-normal mode frequencies [20]. Physically this just indicates that Dirac particles and antiparticles have the same quasi-normal mode spectra in the Schwarzshild black hole spacetime which is quite reasonable here. We shall therefore concentrate just on Eq. (30) with potential $V_{1}$ in evaluating the quasi-normal mode frequencies in the next sections.

\section{QUASI-NORMAL MODE FREQUENCIES FOR THE MASSLESS DIRAC FIELD}

In this section we shall evaluate the quasi-normal frequencies for the massless Dirac field using the WKB approximation. For the massless case, the radial equation (Eq. (30)) is simplified to

$$
\left(-\frac{d^{2}}{d r_{*}^{2}}+V(r, \kappa)\right) \hat{F}=E^{2} \hat{F}
$$

where

$$
V(r, \kappa)=\frac{|\kappa| \Delta^{1 / 2}}{r^{4}}\left[|\kappa| \Delta^{1 / 2}-(r-3)\right]
$$

$r_{*}=r+2 \ln (r / 2-1)$, and $\Delta=r(r-2)$. Note that we have written $V_{1}$ as $V$ because we shall not work with $V_{2}$, which will give the same spectrum of quasi-normal mode frequencies, as discussed in the last section. Also we have used the mass $M$ of the black hole as a unit of mass and length to simplify the notation.

The effective potential $V(r, \kappa)$, which depends only on the absolute value of $\kappa$, is in the form of a barrier. As shown in Fig. 1, the peak of the barrier gets higher and higher as $|\kappa|$ increases. From Fig. 2, we see that the position of the peak

$$
r_{\max }(|\kappa| \rightarrow \infty) \rightarrow 3
$$

and the maximum values of the potential barrier increase indefinitely with $|\kappa|$. In fact,

$$
V\left(|\kappa| \rightarrow \infty, r_{\max }\right)=\frac{\kappa^{2}}{27}
$$

as can be easily derived from Eq. (37). 


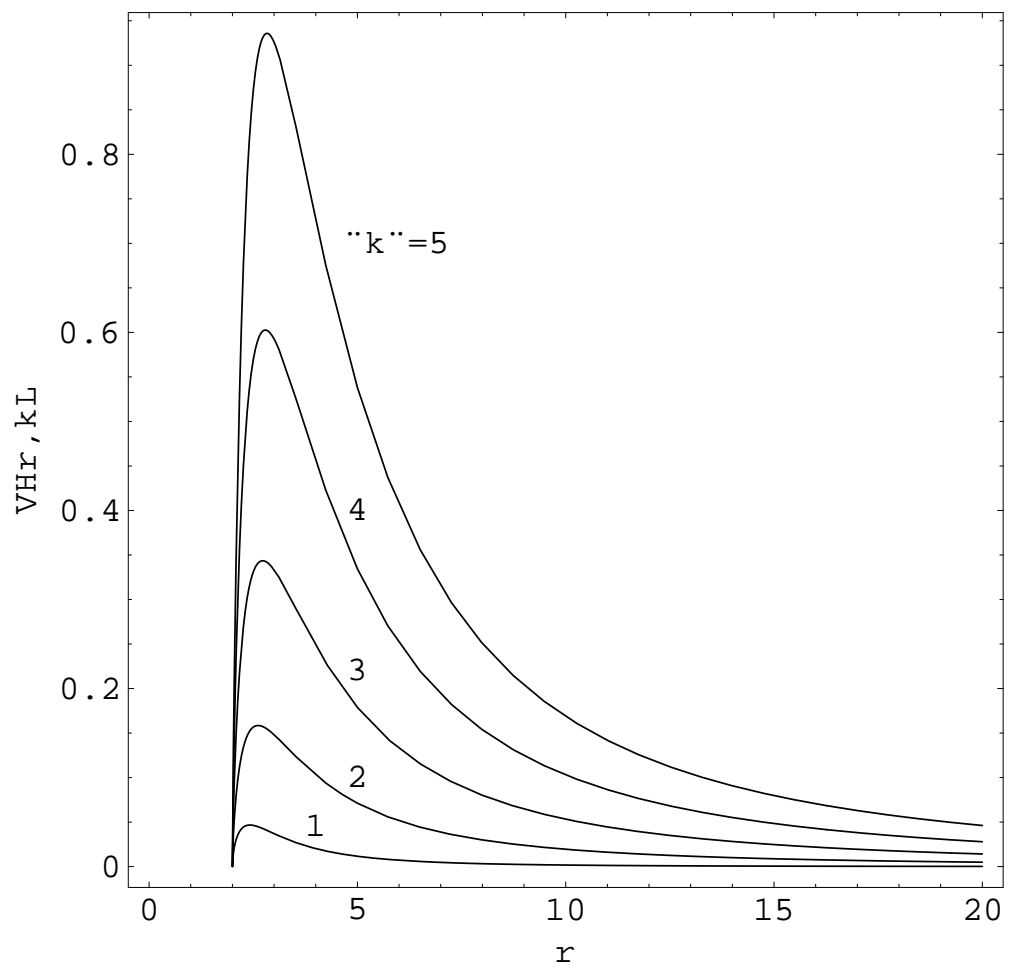

FIG. 1: Variation of the effective potential for the massless Dirac field with $|\kappa|$.
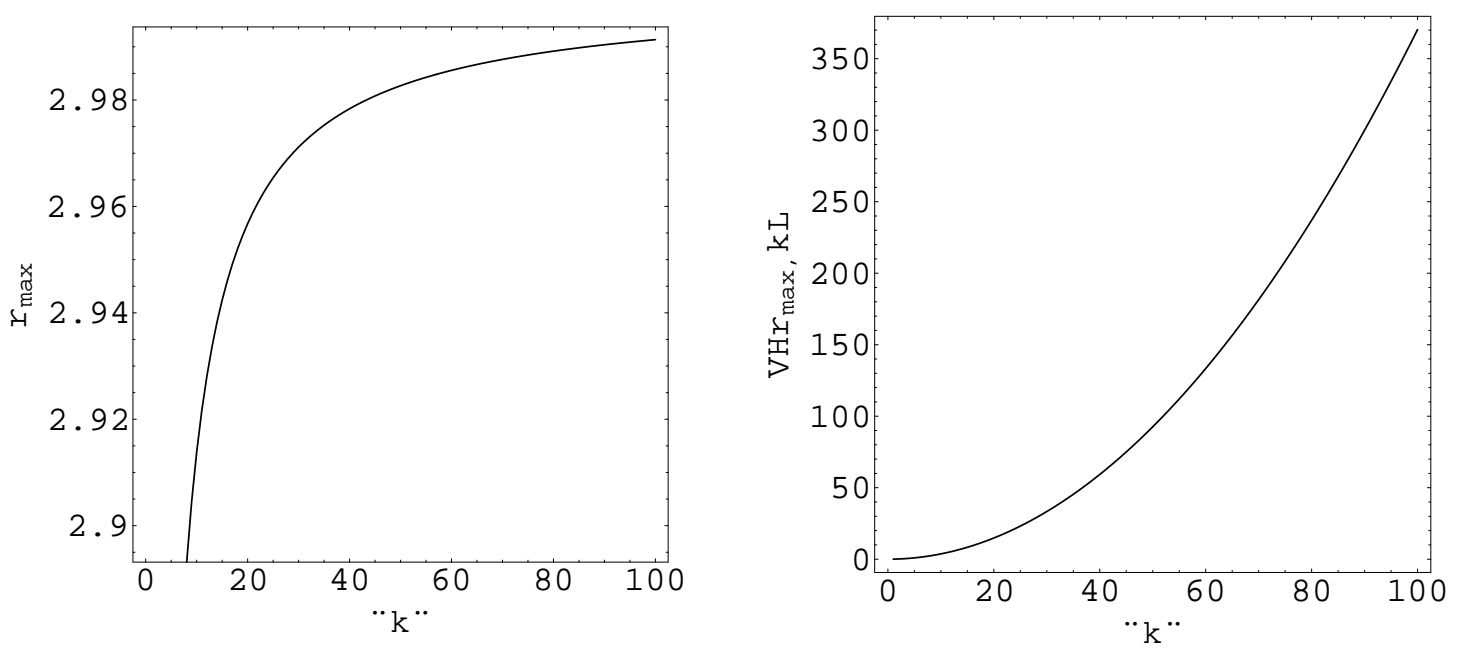

FIG. 2: Variation of the positions of the peaks (left) and the peak values (right) of the effective potential for the massless Dirac field with $|\kappa|$.

To evaluate the quasi-normal mode frequencies, we adopt the WKB approximation devel- 
TABLE I: Massless Dirac quasi-normal mode frequencies.

\begin{tabular}{lllr}
\hline \hline$|\kappa|$ & $n$ & $\operatorname{Re}(E)$ & $\operatorname{Im}(E)$ \\
\hline 1 & 0 & 0.176 & -0.100 \\
2 & 0 & 0.379 & -0.097 \\
& 1 & 0.354 & -0.299 \\
3 & 0 & 0.574 & -0.096 \\
& 1 & 0.556 & -0.293 \\
& 2 & 0.527 & -0.497 \\
4 & 0 & 0.767 & -0.096 \\
& 1 & 0.754 & -0.291 \\
& 2 & 0.730 & -0.491 \\
& 3 & 0.700 & -0.696 \\
& 0 & 0.960 & -0.096 \\
& 1 & 0.950 & -0.290 \\
& 2 & 0.930 & -0.488 \\
& 3 & 0.904 & -0.689 \\
& 4 & 0.872 & -0.894 \\
\hline \hline
\end{tabular}

oped by Schutz, Will, and Iyer [13, 14, 15]. This analytic method has been used extensively in various black hole cases. Comparing with other numerical results, this method has been found to be accurate up to around $1 \%$ for both the real and the imaginary parts of the frequencies for low-lying modes with $n<l$, where $n$ is the mode number and $l$ is the angular momentum quantum number. The formula for the complex quasi-normal mode frequencies $E$ in the WKB approximation, carried to third order beyond the eikonal approximation, is given by [14]

$$
E^{2}=\left[V_{0}+\left(-2 V_{0}^{\prime \prime}\right)^{1 / 2} \Lambda\right]-i\left(n+\frac{1}{2}\right)\left(-2 V_{0}^{\prime \prime}\right)^{1 / 2}(1+\Omega)
$$

where

$$
\begin{aligned}
& \Lambda=\frac{1}{\left(-2 V_{0}^{\prime \prime}\right)^{1 / 2}}\left\{\frac{1}{8}\left(\frac{V_{0}^{(4)}}{V_{0}^{\prime \prime}}\right)\left(\frac{1}{4}+\alpha^{2}\right)-\frac{1}{288}\left(\frac{V_{0}^{\prime \prime \prime}}{V_{0}^{\prime \prime}}\right)^{2}\left(7+60 \alpha^{2}\right)\right\}, \\
& \Omega=\frac{1}{\left(-2 V_{0}^{\prime \prime}\right)}\left\{\frac{5}{6912}\left(\frac{V_{0}^{\prime \prime \prime}}{V_{0}^{\prime \prime}}\right)\left(77+188 \alpha^{2}\right)-\frac{1}{384}\left(\frac{V_{0}^{\prime \prime \prime} V_{0}^{(4)}}{V_{0}^{\prime \prime 3}}\right)\left(51+100 \alpha^{2}\right)\right.
\end{aligned}
$$




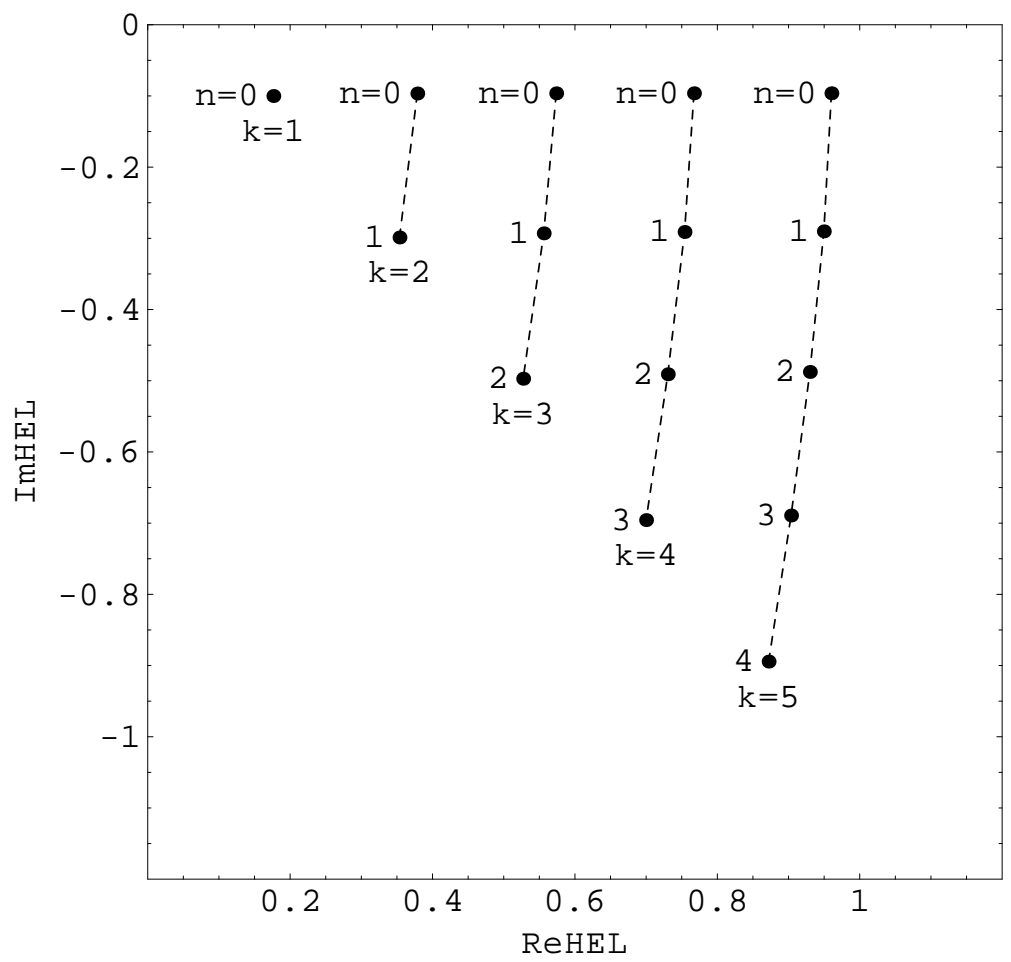

FIG. 3: Massless Dirac quasi-normal mode frequencies

$$
\begin{aligned}
& +\frac{1}{2304}\left(\frac{V_{0}^{(4)}}{V_{0}^{\prime \prime}}\right)^{2}\left(67+68 \alpha^{2}\right)+\frac{1}{288}\left(\frac{V_{0}^{\prime \prime \prime} V_{0}^{(5)}}{V_{0}^{\prime 2}}\right)\left(19+28 \alpha^{2}\right) \\
& \left.-\frac{1}{288}\left(\frac{V_{0}^{(6)}}{V_{0}^{\prime \prime}}\right)\left(5+4 \alpha^{2}\right)\right\} .
\end{aligned}
$$

Here

$$
\begin{aligned}
& \alpha=n+\frac{1}{2}, n=\left\{\begin{array}{l}
0,1,2, \cdots, \operatorname{Re}(E)>0 \\
-1,-2,-3, \cdots, \operatorname{Re}(E)<0,
\end{array}\right. \\
& V_{0}^{(n)}=\left.\frac{d^{n} V}{d r_{*}^{n}}\right|_{r_{*}=r_{*}\left(r_{\max }\right)} .
\end{aligned}
$$

Plugging the effective potential in Eq. (37) into the formula above, we obtain the complex quasi-normal mode frequencies for the massless Dirac field. The values for $0 \leq n<\kappa$ are listed in Table The values for negative $n$ are related to those with positive $n$ by a reflection of the imaginary axis so we do not list them out here. The quasi-normal mode frequencies for positive $\kappa$ are plotted in Fig. 3. The variations of the mode frequencies here are similar to those with integral spin fields in the Schwarzschild black hole spacetime 15]. The real part 
$\operatorname{Re}(E)$ of the frequencies decreases as the mode number $n$ increases for the same angular momentum number $\kappa$. On the other hand, the magitude of the imaginary part $\operatorname{Im}(E)$ of the frequencies increases with $n$. This indicates that the quasi-normal modes with higher mode numbers decay faster than the low-lying ones. Hence, the low-lying quasi-normal modes, possibly with different values of $|\kappa|$, are most relevant to the description of the evolution of a massless Dirac field in the Schwarzschild black hole spacetime.

\section{QUASI-NORMAL MODE FREQUENCIES FOR THE MASSIVE DIRAC FIELD}

In this section we shall evaluate the quasi-normal mode frequencies of the massive Dirac field. Again we start with the radial equation (Eq. (301)),

$$
\left(-\frac{d^{2}}{d \hat{r}_{*}^{2}}+V(r, \kappa, m, E)\right) \hat{F}=E^{2} \hat{F},
$$

where $\hat{r}_{*}=r+2 \ln (r / 2-1)+(1 / 2 E) \tan ^{-1}(m r / \kappa)$, and

$$
\begin{aligned}
& V(r, \kappa, m, E) \\
= & \frac{\Delta^{1 / 2}\left(\kappa^{2}+m^{2} r^{2}\right)^{3 / 2}}{\left(r^{2}\left(\kappa^{2}+m^{2} r^{2}\right)+m \kappa \Delta / 2 E\right)^{2}}\left[\Delta^{1 / 2}\left(\kappa^{2}+m^{2} r^{2}\right)^{3 / 2}+\left((r-1)\left(\kappa^{2}+m^{2} r^{2}\right)+3 m^{2} r \Delta\right)\right] \\
& \quad-\frac{\Delta^{3 / 2}\left(\kappa^{2}+m^{2} r^{2}\right)^{5 / 2}}{\left(r^{2}\left(\kappa^{2}+m^{2} r^{2}\right)+m \kappa \Delta / 2 E\right)^{3}}\left[2 r\left(\kappa^{2}+m^{2} r^{2}\right)+2 m^{2} r^{3}+m \kappa(r-1) / E\right] .
\end{aligned}
$$

Note that the effective potential depends not only on $m$ but also on the energy $E$. This will complicate our evaluation of the quasi-normal mode frequencies using the WKB approximation formula in Eq. (40) because there are $E$ dependence on both sides of the equation.

\section{A. Properties of the effective potential}

Here we analyse the dependence of the effective potential on the parameters $m, \kappa$, and $E$. First, its dependence on $m$ is showed in Fig. [ With energy $E=1$ and with $\kappa=1,2,-1$ and -2 . For small values of $m$, the potential is still in the form of a barrier, but with the asymptotic value

$$
V(r \rightarrow \infty)=m^{2} .
$$

As $m$ is increased, the peak of the potential also increases but does so very slowly. Eventually, the height of the peak is lower than the asymptotic value $m^{2}$. In this case there will be no 

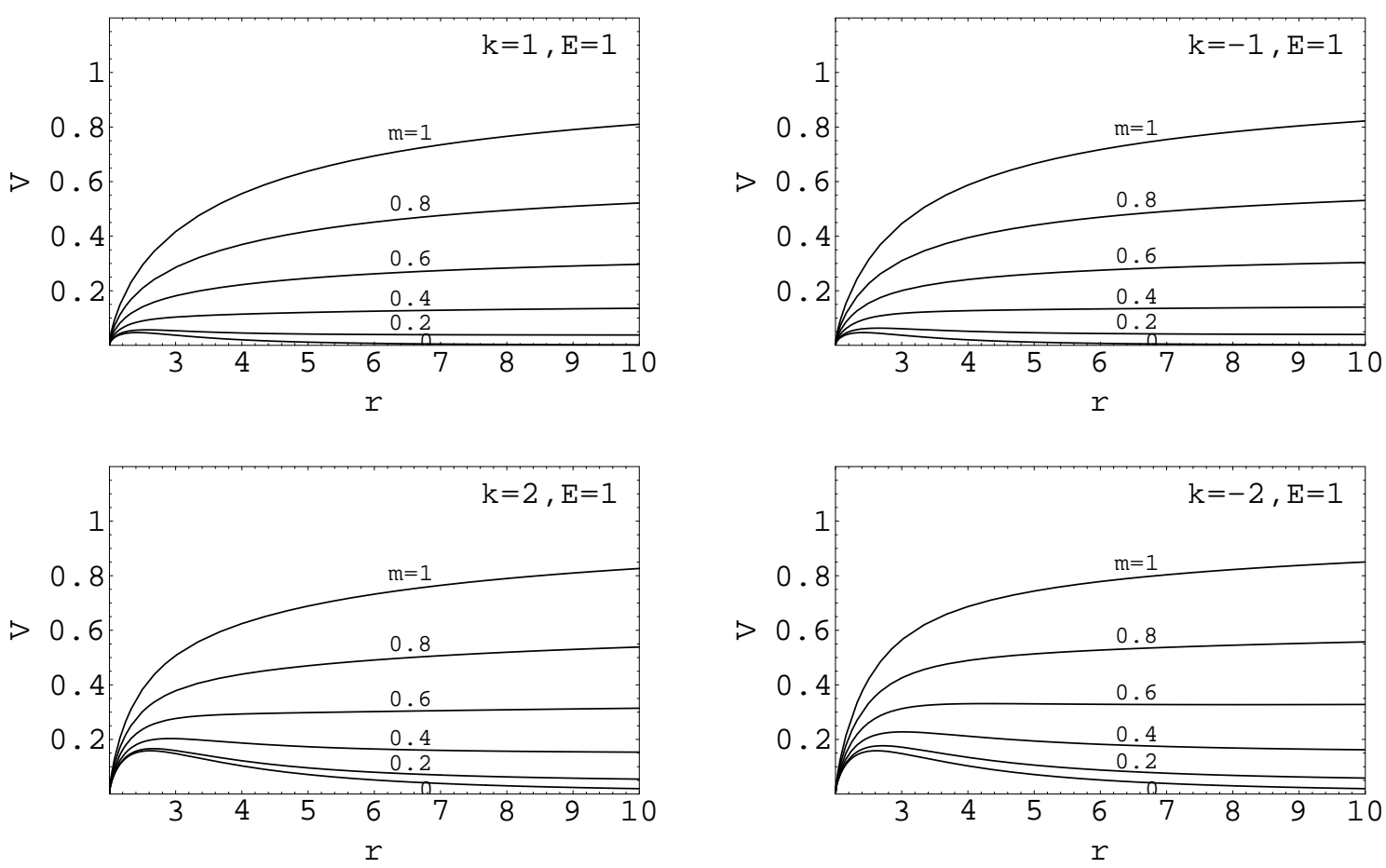

FIG. 4: Variation of the effective potential for the Dirac field with mass $m$ for $\kappa=1,2,-1$ and $-2$.

quasi-normal modes because for tunneling to occur $E^{2}$ of the Dirac field must be smaller than the peak value of the potential. This cannot happen since the energy of the Dirac field is always larger than its mass $m$. When $m$ is increased further, the peak disappears all together, and the potential barrier turns effectively into a potential step. Note that there is a caveat here. The energy $E$ is unknown at the start of the calculation (here we have taken a typical value $E=1$ ) and has to be determined self-consistently. Hence, Fig. 4 (similarly for the other figures below) should only be taken as indicative of the general behaviors of the effective potential $V$.

The effective potential for the massive Dirac field also depends on the energy $E$, and we show its dependence in Fig. 5. $E$ is varied from its minimum value $m$. For positive $\kappa$, the value of the potential increases with $E$, while for negative $\kappa$, it decreases. The variations become smaller and smaller, and we can see from the figure that the potentials are nearly indistingusable for $E=5$ and $E=100$ or higher. The general behaviors of the potential remain the same for all these values of $E$. Indeed, from the form of the potential in Eq. (46)), $E$ appears all in the denominators. The terms involving $E$ can never get large enough to 

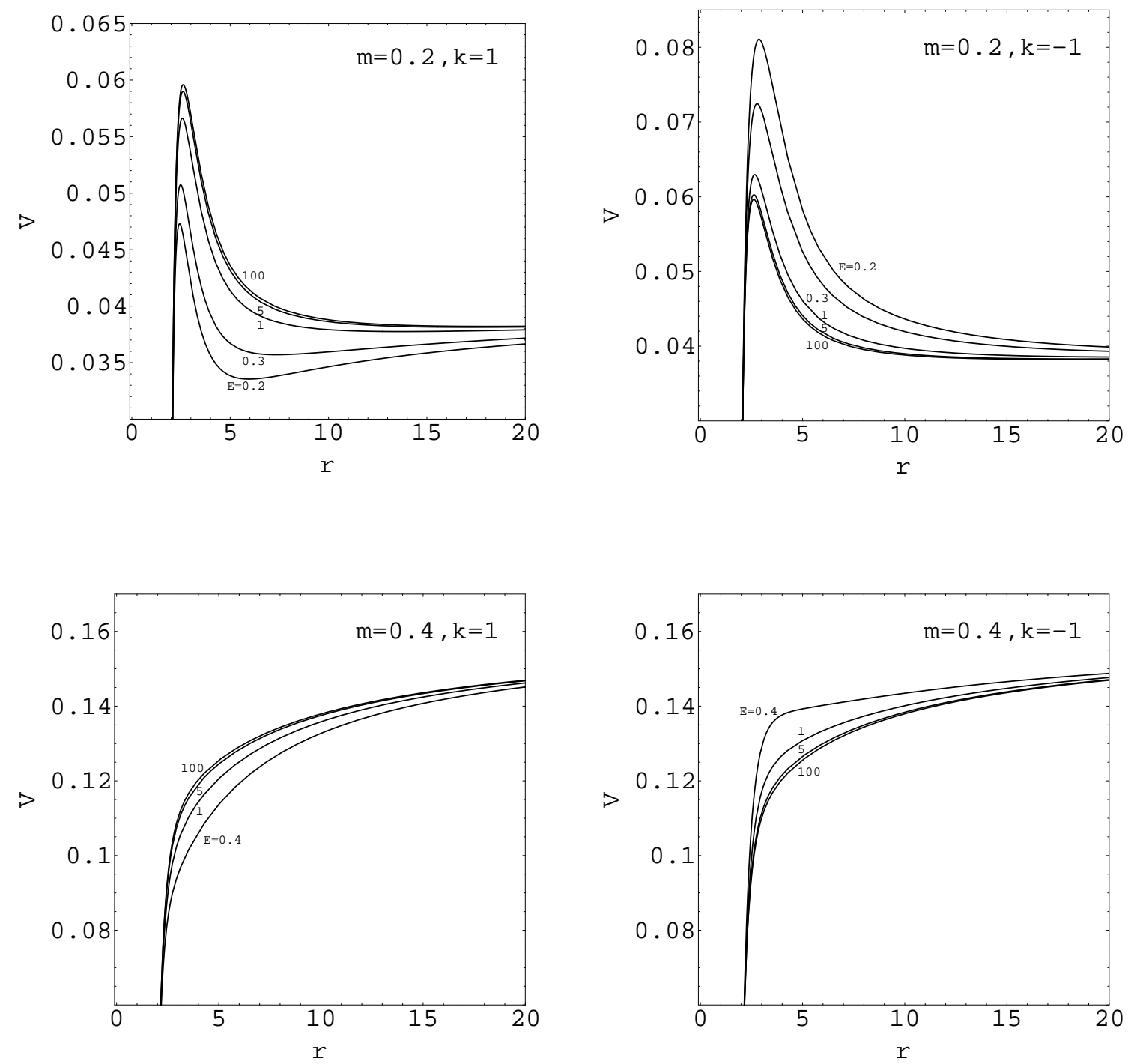

FIG. 5: Variation of the effective potential for the Dirac field with energy $E$ for $m=0.2$ and 0.4 , and $\kappa=1$ and -1 .

change the general behaviors of the potential since $E$ cannot be smaller than $m$.

From the dependences of the potential with $m$ and $E$, we can also estimate the maximum values of $m$ above which quasi-normal modes cannot occur. From the discussions above, we see that quasi-normal modes exist only when (i) the peak value of the potential $V\left(r=r_{\max }\right)$ is larger than $m^{2}$, and (ii) $E^{2}$ of the field is smaller than this peak value. Therefore, one can 
TABLE II: Estimation of the maximum values of the mass $m$ and $\mu(=m / \kappa)$ of the Dirac field above which quasi-normal modes cannot occur.

\begin{tabular}{ccccc}
\hline \hline$|\kappa|$ & $m_{\max }($ positive $\kappa)$ & $\mu_{\max }($ positive $\kappa)$ & $m_{\max }($ negative $\kappa)$ & $\mu_{\max }($ negative $\kappa)$ \\
\hline 1 & 0.224 & 0.224 & 0.333 & -0.333 \\
2 & 0.453 & 0.226 & 0.572 & -0.286 \\
3 & 0.696 & 0.232 & 0.819 & -0.273 \\
4 & 0.944 & 0.236 & 1.067 & -0.267 \\
5 & 1.192 & 0.238 & 1.316 & -0.263 \\
\hline \hline
\end{tabular}
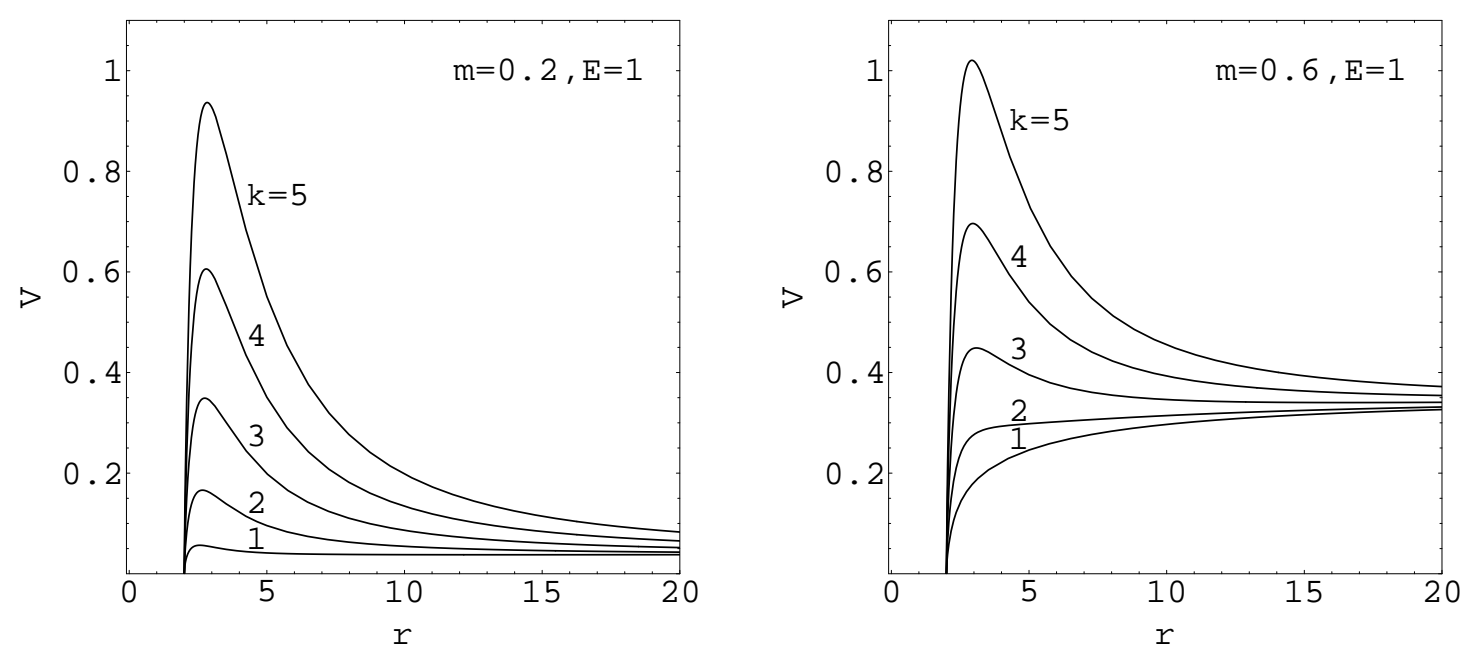

FIG. 6: Variation of the effective potential for the Dirac field with $\kappa$ for $m=0.2$ and 0.6 .

estimate this maximum value $m_{\max }$ from the relation

$$
V\left(r_{\max }, m_{\max }, \kappa, E=m_{\max }\right)=\left(m_{\max }\right)^{2}
$$

which can be solved numerically. The result is tabulated in Table III where we have also given the maximum values of $\mu=m / \kappa$ which will be useful in the calculation below.

Finally, in Fig. [6] we show the dependence of the effective potential with the angular momentum quantum number $\kappa$. We see that as $\kappa$ increases, the behaviors of the potential approach to that of the massless one, as shown in Fig. 1, regardless the mass of the field. 
This can be understood by writing the potential in Eq. (46) as

$$
\begin{aligned}
& V(r, \kappa, m=\kappa \mu, E) \\
= & \frac{|\kappa| \Delta^{1 / 2}\left(1+\mu^{2} r^{2}\right)^{3 / 2}}{\left(r^{2}\left(1+\mu^{2} r^{2}\right)+\mu \Delta / 2 E\right)^{2}}\left[|\kappa| \Delta^{1 / 2}\left(1+\mu^{2} r^{2}\right)^{3 / 2}+\left((r-1)\left(1+\mu^{2} r^{2}\right)+3 \mu^{2} r \Delta\right)\right] \\
& \quad-\frac{|\kappa| \Delta^{3 / 2}\left(1+\mu^{2} r^{2}\right)^{5 / 2}}{\left(r^{2}\left(1+\mu^{2} r^{2}\right)+\mu \Delta / 2 E\right)^{3}}\left[2 r\left(1+\mu^{2} r^{2}\right)+2 \mu^{2} r^{3}+\mu(r-1) / E\right],
\end{aligned}
$$

where $\mu$ is as defined above. The dependence of mass is all through $\mu$. As $|\kappa| \rightarrow \infty, \mu \rightarrow 0$ regardless the value of $m$, and the effective potential will approach to that of the massless one. Also from Table $\llbracket$ the maximum magnitudes of $\mu$ are around $0.2 \sim 0.3$ regardless the value of $\kappa . \mu$ can thus be used as a small parameter for expansions as we shall do below.

\section{B. Evaluation of the quasi-normal mode frequencies}

The evaluation of the massive Dirac quasi-normal mode frequencies is more complicated than the massless ones due to the presence of the energy dependence on both sides of the Eq. (40). Since the maximum magnitudes of $\mu$ are only around $0.2 \sim 0.3$, we can try to obtain the quasi-normal mode frequencies in the WKB approximation as power series of $\mu$ for given values of $\kappa$ similar to the power series expansions in [21] and [22].

We first express the position of the peak of the effective potential as a series up to order $\mu^{6}$

$$
\begin{aligned}
r_{\max } & =r_{0}+r_{1} \mu+r_{2} \mu^{2}+r_{3} \mu^{3}+r_{4} \mu^{4}+r_{5} \mu^{5}+r_{6} \mu^{6} \\
& =r_{0}+\Sigma
\end{aligned}
$$

by requiring

$$
\begin{aligned}
0= & V^{\prime}\left(r_{\max }\right) \\
= & V^{\prime}\left(r_{0}\right)+\Sigma V^{\prime \prime}\left(r_{0}\right)+\frac{1}{2} \Sigma^{2} V^{\prime \prime \prime}\left(r_{0}\right)+\frac{1}{6} \Sigma^{3} V^{(4)}\left(r_{0}\right) \\
& +\frac{1}{24} \Sigma^{4} V^{(5)}\left(r_{0}\right)+\frac{1}{120} \Sigma^{5} V^{(6)}\left(r_{0}\right)+\frac{1}{720} \Sigma^{6} V^{(7)}\left(r_{0}\right) .
\end{aligned}
$$

Since $r_{0}$ is just the position of the peak for the massless case obtained in Section III, we can evaluate the coefficients $r_{i}$ 's order by order from this equation. For example, for $|\kappa|=1$, we obtain

$$
r_{\max }=2.42-\mu\left(\frac{0.182}{E}\right)+\mu^{2}\left(3.66+\frac{0.0755}{E^{2}}\right)-\mu^{3}\left(\frac{1.07}{E}+\frac{0.0322}{E^{3}}\right)
$$




$$
\begin{aligned}
+ & \mu^{4}\left(18.8-\frac{0.252}{E^{2}}+\frac{0.0142}{E^{4}}\right)-\mu^{5}\left(\frac{0.526}{E}-\frac{0.624}{E^{3}}+\frac{0.00638}{E^{5}}\right) \\
& +\mu^{6}\left(117-\frac{8.16}{E^{2}}-\frac{0.597}{E^{4}}+\frac{0.00293}{E^{6}}\right) .
\end{aligned}
$$

The coefficients in the above expansion involve the unknown $E$. We can likewise expand $E$,

$$
E=E_{0}+E_{1} \mu+E_{2} \mu^{2}+E_{3} \mu^{3}+E_{4} \mu^{4}+E_{5} \mu^{5}+E_{6} \mu^{6} .
$$

We plug this expansion back into Eq. (52), and expand again up to order $\mu^{6}$. Using this new expansion for $r_{\max }$, we can then expand the derivative of the potential $V_{0}^{(n)}$. Note that the derivatives are performed with respect to $\hat{r}_{*}$, and

$$
\begin{aligned}
\frac{d}{d \hat{r}_{*}}= & {\left[\frac{1}{1-2 / r}+\frac{\mu}{2 E\left(1+\mu^{2} r^{2}\right)}\right]^{-1} \frac{d}{d r} } \\
= & {\left[\left(1-\frac{2}{r}\right)-\frac{1}{2 E}\left(1-\frac{2}{r}\right)^{2} \mu+\frac{1}{4 E^{2}}\left(1-\frac{2}{r}\right)^{3} \mu^{2}\right.} \\
& -\frac{1}{8 E^{3} r^{2}}\left(1-\frac{2}{r}\right)^{2}\left(4-4 r+r^{2}-4 E^{2} r^{4}\right) \mu^{3} \\
+ & \frac{1}{16 E^{4} r^{2}}\left(1-\frac{2}{r}\right)^{3}\left(4-4 r+r^{2}-8 E^{2} r^{4}\right) \mu^{4} \\
& -\frac{1}{32 E^{5} r^{4}}\left(1-\frac{2}{r}\right)^{3}\left(16-32 r+24 r^{2}-8 r^{3}\right. \\
& \left.+\left(1-48 E^{2}\right) r^{4}+48 E^{2} r^{5}-12 E^{2} r^{6}+16 E^{4} r^{8}\right) \mu^{5} \\
& +\frac{1}{64 E^{6} r^{4}}\left(1-\frac{2}{r}\right)^{3}\left(16-32 r+24 r^{2}-8 r^{3}\right. \\
& \left.\left.+\left(1-64 E^{2}\right) r^{4}+64 E^{2} r^{5}-16 E^{2} r^{6}+48 E^{4} r^{8}\right) \mu^{6}+\cdots\right] \frac{d}{d r} .
\end{aligned}
$$

We put all these expansions back to Eq. (40) and solve the coefficients $E_{i}$ 's self-consistently order by order in $\mu$. The results are tabulated in Tables III and IV.

With these coefficients we can evaluate the quasi-normal mode frequencies by putting in the value of $\mu$ for both positive and negative values of $\kappa$. From Table the maximum value of $\mu$ that could have quasi-normal modes is 0.224 for $\kappa=1$ and increases to 0.238 for $\kappa=5$. In fact, the value will increase for larger values of $\kappa$, but converges to the value $\sim 0.25$. Similarly for the variation of $\mu_{\max }$ with negative $\kappa$, the magnitude decreases from 0.333 for $\kappa=-1$ to 0.263 for $\kappa=-5$, and will converge to $\sim 0.25$ for large values of $\kappa$. Therefore, we shall try to evaluate the frequencies for $|\mu|$ from 0 to 0.2 .

First we examine the frequencies for $\kappa=1$ and $n=0$. For $m=0$, we have

$$
E=E_{0}=0.176-0.100 i,
$$


TABLE III: Real parts of the coefficients of the expansions in powers of $\mu$ for the quasi-normal mode frequencies with $|\kappa|=1$ to 5 .

\begin{tabular}{ccccccccc}
\hline \hline$|\kappa|$ & $n$ & $\operatorname{Re}\left(E_{0}\right)$ & $\operatorname{Re}\left(E_{1}\right)$ & $\operatorname{Re}\left(E_{2}\right)$ & $\operatorname{Re}\left(E_{3}\right)$ & $\operatorname{Re}\left(E_{4}\right)$ & $\operatorname{Re}\left(E_{5}\right)$ & $\operatorname{Re}\left(E_{6}\right)$ \\
\hline 1 & 0 & 0.176 & -0.168 & 0.521 & -0.393 & -0.00367 & -3.13 & 74.2 \\
2 & 0 & 0.379 & -0.166 & 1.49 & 0.158 & 1.01 & -0.728 & -0.845 \\
& 1 & 0.354 & -0.136 & 0.478 & -0.510 & -3.81 & 9.23 & -24.0 \\
3 & 0 & 0.574 & -0.166 & 2.43 & 0.335 & 1.84 & 0.721 & 2.75 \\
& 1 & 0.556 & -0.152 & 1.55 & -0.366 & -1.92 & -3.30 & -8.91 \\
& 2 & 0.527 & -0.129 & 0.463 & -0.455 & -5.84 & 16.6 & -93.0 \\
4 & 0 & 0.767 & -0.166 & 3.34 & 0.404 & 2.56 & 1.13 & 6.85 \\
& 1 & 0.754 & -0.158 & 2.61 & -0.115 & 0.291 & -2.31 & -2.43 \\
& 2 & 0.730 & -0.144 & 1.50 & -0.494 & -5.64 & -0.255 & -49.8 \\
& 3 & 0.700 & -0.126 & 0.460 & -0.427 & -7.39 & 20.8 & -152 \\
& 0 & 0.960 & -0.166 & 4.23 & 0.437 & 3.24 & 1.28 & 9.98 \\
& 0.950 & -0.161 & 3.62 & 0.0610 & 1.80 & -0.952 & 2.32 \\
& 1 & 0.950 & -0.152 & 2.60 & -0.358 & -3.02 & -3.28 & -23.4 \\
& 2 & 0.930 & -0.138 & 1.46 & -0.514 & -8.82 & 4.03 & -103 \\
& 0.904 & -0.124 & 0.462 & -0.408 & -8.81 & 23.5 & -204 \\
\hline \hline
\end{tabular}

which is the massless case we have obtained in the last section.

For $m=0.1$, we have

$$
\begin{aligned}
E_{0} & =0.176-0.100 i \\
E_{1} \mu & =-0.0168-0.00711 i \\
E_{2} \mu^{2} & =0.00521+0.00452 i \\
E_{3} \mu^{3} & =-0.000393+0.000375 i \\
E_{4} \mu^{4} & =-0.00000367+0.000138 i \\
E_{5} \mu^{5} & =-0.0000313-0.0000859 i, \\
E_{6} \mu^{6} & =0.0000742-0.0000140 i,
\end{aligned}
$$


TABLE IV: Imaginary parts of the coefficients of the expansions in powers of $\mu$ for the quasi-normal mode frequencies with $|\kappa|=1$ to 5 .

\begin{tabular}{ccccccccc}
\hline \hline$|\kappa|$ & $n$ & $\operatorname{Im}\left(E_{0}\right)$ & $\operatorname{Im}\left(E_{1}\right)$ & $\operatorname{Im}\left(E_{2}\right)$ & $\operatorname{Im}\left(E_{3}\right)$ & $\operatorname{Im}\left(E_{4}\right)$ & $\operatorname{Im}\left(E_{5}\right)$ & $\operatorname{Im}\left(E_{6}\right)$ \\
\hline 1 & 0 & -0.100 & -0.0711 & 0.452 & 0.375 & 1.38 & -8.59 & -14.0 \\
2 & 0 & -0.0965 & -0.0282 & 0.653 & 0.480 & 1.38 & 1.59 & -3.47 \\
& 1 & -0.299 & -0.0771 & 1.16 & -0.0876 & -5.43 & -16.9 & 7.54 \\
3 & 0 & -0.0963 & -0.0185 & 0.690 & 0.368 & 1.21 & 1.46 & 4.76 \\
& 1 & -0.293 & -0.0533 & 1.64 & 0.391 & 1.38 & -4.30 & 22.6 \\
& 2 & -0.497 & -0.0770 & 1.72 & -0.172 & -13.3 & -18.9 & 37.7 \\
4 & 0 & -0.0963 & -0.0139 & 0.704 & 0.291 & 1.13 & 1.14 & 6.02 \\
& 1 & -0.291 & -0.0406 & 1.85 & 0.516 & 2.87 & 0.442 & 21.0 \\
& 2 & -0.491 & -0.0627 & 2.38 & 0.186 & -2.21 & -11.0 & 59.4 \\
& 3 & -0.696 & -0.0771 & 2.27 & -0.204 & -20.5 & -19.6 & 52.6 \\
5 & 0 & -0.0963 & -0.0111 & 0.710 & 0.239 & 1.08 & 0.914 & 6.23 \\
& 1 & -0.290 & -0.0327 & 1.96 & 0.517 & 3.20 & 1.60 & 19.9 \\
\hline \hline & & -0.488 & -0.0521 & 2.76 & 0.399 & 2.25 & -4.00 & 53.6 \\
& & -0.689 & -0.0672 & 3.01 & 0.0573 & -7.44 & -15.0 & 81.5 \\
& & -0.894 & -0.0773 & 2.80 & -0.223 & -27.5 & -19.8 & 53.6 \\
\hline
\end{tabular}

and

$$
E=0.164-0.102 i
$$

Hence, if we take three significant figures, both the real and the imaginary parts of $E_{3} \mu^{3}$ and higher order terms do not contribute to the frequency. The result in Eq. (57) is thus accurate up to at least three significant figures.

For $m=0.2$, we have

$$
\begin{aligned}
E_{0} & =0.176-0.100 i \\
E_{1} \mu & =-0.0337-0.0142 i \\
E_{2} \mu^{2} & =0.0208+0.0181 i \\
E_{3} \mu^{3} & =-0.00315+0.00300 i \\
E_{4} \mu^{4} & =-0.00000587+0.00221 i
\end{aligned}
$$




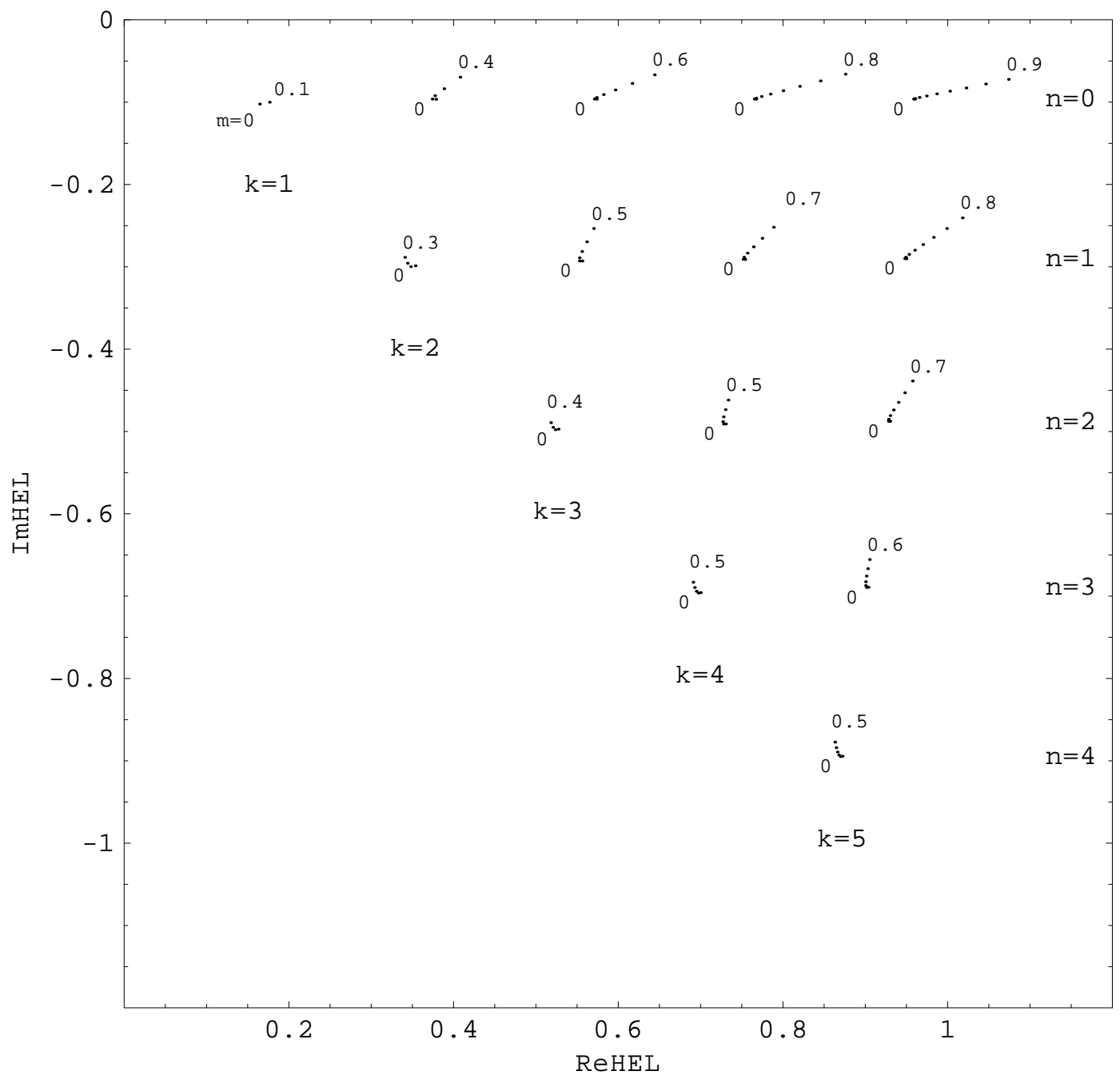

FIG. 7: Massive Dirac quasi-normal mode frequencies

$$
\begin{aligned}
& E_{5} \mu^{5}=-0.00100-0.00275 i, \\
& E_{6} \mu^{6}=0.00475-0.000893 i
\end{aligned}
$$

and

$$
E=0.164-0.0947 i
$$

Here we see that even the highest order term $E_{6} \mu^{6}$ will contribute to both the real and the imaginary parts of $E$. If we require our result to be accurate up to three significant figures, we need to go to orders higher than $\mu^{6}$. However, we have only expanded terms up to $\mu^{6}$, we are thus forced to disregard this result for $m=0.2$, even though there should be a quasi-normal mode around that value. 
In summary, for different values of $\kappa$ and $n$, we increase the values of $m$ (or $\mu$ ) from 0 up to the point that three significant figure accuracy cannot be maintained with terms up to only $\mu^{6}$, and then we stop at that value. The results for $\kappa=1$ to 5 are plotted in Fig. 7 . Comparing with Table【, we see that the largest value of $m$ for each $\kappa$ and $n$ that we present in Fig. 7 is in fact very close to the maximum value $m_{\max }$. On the other hand, the trends of the variations of $E$ with $m$ are similar for negative $\kappa$ so we do not plot them out.

As we can see from Fig. [7, the real parts of the quasi-normal mode frequencies in general increase with the mass of the field. This can be understood from the fact that when the mass of the field increases, the peak of the effective potential gets higher. The real part of the energy should therefore increase. We also see that the magnitudes of the imaginary parts decrease with the mass. We thus expect Dirac fields with larger masses (same $\kappa$ and $n$ ) to decay slower during their evolutions.

\section{CONCLUSIONS AND DISCUSSIONS}

We have evaluated both the massless and the massive Dirac quasi-normal mode frequencies in the Schwarzschild black hole spacetime using the WKB approximation. Only the cases with $\kappa>n$, where $\kappa$ is the total angular momentum number and $\mathrm{n}$ is the mode number, are considered since these are the cases known to be accurate for the WKB approximation with the integral spin fields [15]. For the massless Dirac field, we see that the real parts of the quasi-normal mode frequencies increase with $\kappa$, but they decrease with $n$ for fixed $\kappa$. As for the imaginary parts, the magnitudes of which increase with $n$. Hence, the fields with $n=0$ will decay most slowly.

For the massive cases, the evaluation of the quasi-normal mode frequencies is complicated by the fact that the effective potential also depends on the energy $E$. A further approximation is needed to carry through the calculation in which we make perturbative expansions for all the relevant quantities in powers of the parameter $\mu=m / \kappa$ where $m$ is the mass of the field. In this way we are able to obtain the numerical values of both the real and the imaginary parts of the mode frequencies up to three significant figures. In general, when the mass of the field is increased, the real parts of the frequencies increase while the magnitudes of the imaginary parts decrease. Fields with higher masses will therefore decay slower. This result is similar to that of the massive scalar field [22]. 
The calculation carried out here can be extended to other black hole spacetimes, for example, the charged Reissner-Nordström black hole or the rotating Kerr black hole. However, for the Kerr black hole, the situation is further complicated by the fact that there is no close form for the effective potential. Furthermore, we can consider dilaton black holes or black holes in the anti-de Sitter space both of which are closely related to string theories.

As we have mentioned ealier, our main concern is about the evolution of Dirac fields in curved spacetimes, especially black hole spacetimes. After calculating the quasi-normal mode frequencies, our next step would be to understand the late-time tail behaviors of the fields. Together with the Green's function method, we should be able to get a more complete picture of the evolution, and hopefully towards a better understanding of the gravitational collapse of Dirac (or fermionic) matter.

\section{Acknowledgments}

This work is supported by the National Science Council of the Republic of China under contract number NSC 90-2112-M-032-004.

[1] F. Finster, J. Smoller, and S.-T. Yau, Phys. Rev. D. 59, 104020 (1999).

[2] F. Finster, J. Smoller, and S.-T. Yau, Phys. Lett. A259, 431 (1999).

[3] F. Finster, J. Smoller, and S.-T. Yau, Commun. Math. Phys. 205, 249 (1999).

[4] F. Finster, J. Smoller, and S.-T. Yau, J. Math. Phys. 41, 2173 (2000).

[5] F. Finster, N. Kamran, J. Smoller, and S.-T. Yau, Commun. Pure Appl. Math. 53, 902 (2000).

[6] F. Finster, J. Smoller, and S.-T. Yau, J. Math. Phys. 41, 3943 (2000).

[7] F. Finster, J. Smoller, and S.-T. Yau, Adv. Theor. Math. Phys. 4, 1231 (2002).

[8] F. Finster, J. Smoller, and S.-T. Yau, gr-qc/0211043.

[9] F. Finster, N. Kamran, J. Smoller, and S.-T. Yau, Commun. Math. Phys. 230, 201 (2002).

[10] F. Finster, N. Kamran, J. Smoller, and S.-T. Yau, gr-qc/0005088.

[11] V. P. Frolov and I. D. Novikov, Black Hole Physics: Basic Concepts and New Developments (Kluwer Academic Publishers, 1998).

[12] K. D. Kokkotas and B. G. Schmidt, Living Rev. Relativity 2 (1999). 
[13] B. F. Schutz and C. M. Will, Astrophys. J. Lett. 291, L33 (1985).

[14] S. Iyer and C. M. Will, Phys. Rev. D. 35, 3621 (1987).

[15] S. Iyer, Phys. Rev. D. 35, 3632 (1987).

[16] D. R. Brill and J. A. Wheeler, Rev. Mod. Phys. 29, 465 (1957).

[17] J. D. Bjorken and S. D. Drell, Relativistic Quantum Mechanics (McGraw Hill, 1964).

[18] S. Chandrasekhar, The Mathematical Theory of Black Holes (Clarendon Press, 1983).

[19] F. Cooper, A. Khare, and U. Sukhatme, Phys. Rept. 251, 267 (1995).

[20] A. Anderson and R. H. Price, Phys. Rev. D. 43, 3147 (1991).

[21] E. Seidel and S. Iyer, Phys. Rev. D. 41, 374 (1990).

[22] L. E. Simone and C. M. Will, Class. Quantum Grav. 9, 963 (1992). 